\title{
Construction and Enumeration of Different Configuration
}

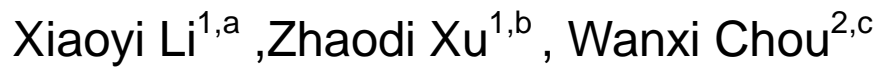

1. School of Mathematics and Systems Science, Shenyang normal University, Liaoning Shenyang 110034.China.

2. School of Civil Engineering and Architectures, Anhui University of Sciences and Technology,

Anhui Huainan.232001, China.

\section{E-mail: lixy@synu.edu.cn}

Keywords: export design; residual design; construction; Isomorphism

\begin{abstract}
This paper clarifies the basic idea of block design on different configuration. Clarify the properties of $(v, k, \lambda)$ derived design and $(v, v-k, v-2 r+\lambda)$ complement design. Introduce the nature of $(v, k, \lambda)$ derived design and $(v, v-k, v-2 r+\lambda)$ complementary design. The method of constructing $(v, k, \lambda)$ derived design and $(v, v-k, v-2 r+\lambda)$ complementary design is given. The entire procedure of constructing $(16,6,2)$ design $(16,10,6)$ design is also presented completely. 11 disjoint $(16,6,2)$ design and 11 disjoint $\quad(16,10,6)$ design are obtained.
\end{abstract}

\section{Introduction}

Block design theory is an important branch of combinatorial mathematics. It plays an important role in the field of experimental design, competition arrangements and digital communication. In 1850, England mathematician Kirkman proposed an interesting problem ' 15 girls question' and solve it in the same year. In 1971, D. K. Ray-Chaudhuri and R. M. Wilson published "the solution of Kirkman 15 girls question”, in order to clarify the structure of Kirkman triple system. More than a century, whether there always exists $6 n+3$ order of Kirkman triple system for each $n=0,1,2, \cdots$ has been a difficult problem [1-5]. In 2003, the author proposes a method for the construction of high order Steiner triple system, and structures $\quad v=27,45,81,135$ order of Kirkman triple system [6, 7].

\section{Basic idea}

Let there be two multiple-element systems with $v$ order, one is $(v, k, \lambda)$ design with the block capacity of $\mathrm{k} S^{\prime}=\left\{B_{1}^{\prime}, B_{2}^{\prime}, \cdots, B_{v}^{\prime}\right\}$ on $X=\{1,2, \cdots, v\}$; The other one is $(v, v-k, v-2 r+\lambda)$ design with the block capacity of $v-k S^{\prime \prime}=\left\{B_{1}^{\prime \prime}, B_{2}^{\prime \prime}, \cdots, B_{v}^{\prime \prime}\right\}$ on $X=\{1,2, \cdots, v\}$. If there exists the following complementary relationship between $v$ blocks $B_{1}^{(1)} \cdot B_{2}^{(1)}, \cdots, B_{v}^{(1)}$ of $(v, k, \lambda)$ design $S^{\prime}=\left\{B_{1}^{\prime}, B_{2}^{\prime}, \cdots, B_{v}^{\prime}\right\} \quad$ and $\quad v \quad$ blocks $\quad B_{1}^{(3)}, B_{2}^{(3)}, \cdots, B_{v}^{(3)} \quad$ of $\quad(v, v-k, v-2 r+\lambda) \quad$ design $S^{\prime \prime}=\left\{B_{1}^{\prime \prime}, B_{2}^{\prime \prime}, \cdots, B_{v}^{\prime \prime}\right\}$ :

$$
B_{1}^{(3)}=X \cap B_{1}^{(1)}, B_{2}^{(3)}=X \cap B_{2}^{(1)}, \cdots, B_{v}^{(3)}=X \cap B_{v}^{(1)}
$$

Then the $S^{\prime}=\left\{B_{1}^{\prime}, B_{2}^{\prime}, \cdots, B_{v}^{\prime}\right\}$ design of $(v, k, \lambda)$ is called derived design; the $S^{\prime \prime}=\left\{B_{1}^{\prime \prime}, B_{2}^{\prime \prime}, \cdots, B_{v}^{\prime \prime}\right\}$ design of $(v, v-k, v-2 r+\lambda)$ is called complementary design.

The derived design $S^{\prime}=\left\{B_{1}^{\prime}, B_{2}^{\prime}, \cdots, B_{v}^{\prime}\right\}$ of $(v, k, \lambda)$ and the complementary design $S^{\prime \prime}=\left\{B_{1}^{\prime \prime}, B_{2}^{\prime \prime}, \cdots, B_{v}^{\prime \prime}\right\}$ of $(v, v-k, v-2 r+\lambda)$ has the following properties.

(1) The order of the derived design $S^{\prime}$ of $(v, k, \lambda)$ equals to the order of the complementary design $S^{\prime \prime}$ of $(v, v-k, v-2 r+\lambda)$. 
(2) The block capacity of the derived design $S^{\prime}$ of $(v, k, \lambda)$ is $k$, the block capacity of the complementary design $S^{\prime}$ of $(v, v-k, v-2 r+\lambda)$ is $v-k$.

(3) There are complementary relationships between derived design $S^{\prime}$ of $(v, k, \lambda)$ and the complementary design of $(v, v-k, v-2 r+\lambda)$ as follows:

$$
B_{1}^{\prime \prime}=X \cap B_{1}^{\prime}, \quad B_{2}^{\prime \prime}=X \cap B_{2}^{\prime}, \ldots, \quad B_{v}^{\prime \prime}=X \cap B_{v}^{\prime}
$$

(4) The number of disjoint in the derived design $S^{\prime}$ of $(v, k, \lambda)$ is the same with the complementary design $S^{\prime}$ of $(v, v-k, v-2 r+\lambda)$.

(5) If the correlation matrix for the derived design $S^{\prime}$ of $(v, k, \lambda)$ is $\mathrm{A}$, then the correlation matrix for the complementary design $S^{\prime}$ of $(v, v-k, v-2 r+\lambda)$ is $J_{v \times k}-A$.

\section{The design and construction of $(16,6,2)$}

The constructed design can be summarized as following step:

(1)Using the subset $X_{1}=\{1,2,3,4,5,6\}$ of $X=\{1,2,3, \cdots, 16\}$ as a variable element set of design $(15,6,5,2,1)$ and the other subset $X_{2}=\{7,8,9, \cdots, 16\}$ as a variable element set of design $(15,10,6,4,2)$.

(2)Construct 15 blocks of design $(15,6,5,2,1)$ on $X_{1}=\{1,2,3,4,5,6\}$ :

$$
B_{1}=(1,2), B_{2}=(1,3), B_{3}=(1,3), B_{4}=(1,4), \ldots, B_{15}=(5,6) .
$$

(3)Construct 15 blocks of design $(15,10,6,4,2)$ on $X_{2}=\{7,8,9, \cdots, 16\}$ :

$$
B_{1}=(7,8,9,10) \quad B_{2}=(7,11,12,13), \quad B_{3}=(8,11,14,15), \ldots, \quad B_{15}=(7,8,13,14) .
$$

(4)Give the union of the block $B_{1}^{\prime}, B_{2}^{\prime}, \cdots, B_{15}^{\prime}$ from design $(15,6,5,2,1)$ and $B_{1}^{\prime \prime}, B_{2}^{\prime \prime}, \cdots, B_{15}^{\prime \prime}$ from design $(15,10,6,4,2)$. And let $B_{1}=X_{1}=\{1,2,3,4,5,6\}$ and other blocks $B_{2}=B_{1}^{\prime} \cup B_{1}^{\prime \prime}$, $B_{3}=B_{2}^{\prime} \cup B_{2}^{\prime \prime}, B_{4}=B_{3}^{\prime} \cup B_{3}^{\prime \prime}, \ldots, B_{16}=B_{15}^{\prime} \cup B_{15}^{\prime \prime}$. Then we can obtain the design $S^{\prime}=\left\{B_{1}^{\prime}, B_{2}^{\prime}, \cdots, B_{16}^{\prime}\right\}$ of $(16,6,2)$ as follows:

$$
\begin{array}{cccc}
S_{1}^{\prime}=\{(1,2,3,4,5,6), \quad(1,2,7,8,9,10), & (1,3,7,11,12,13), \quad(1,4,8,11,14,15), & (1,5,9,12,14,16), \\
(1,6,10,13,15,16), \quad(2,3,7,14,15,16), & (2,4,8,12,13,16), \quad(2,5,9,11,13,15), \quad(2,6,10,11,12,14), \\
(3,4,9,10,13,14), \quad(3,5,8,10,11,16), & (3,6,8,9,12,15), \quad(4,5,7,10,12,15), \quad(4,6,7,9,11,16), \\
(5,6,7,8,13,14)\} 。
\end{array}
$$

Perform the above construction steps and obtain other ten different configurations of design $(16,6,2) \quad S_{2}^{\prime}, S_{3}^{\prime}, \ldots, S_{11}^{\prime}$, as follows:

$S_{2}^{\prime}=\{(1,2,5,6,7,8), \quad(1,2,3,4,9,10), \quad(1,3,5,11,12,13), \quad(1,4,6,11,14,15), \quad(1,7,9,12,14,16)$, $(1,8,10,13,15,16),(2,3,5,14,15,16),(2,4,6,12,13,16),(2,7,9,11,13,15),(2,8,10,11,12,14)$, $(3,4,7,8,9,15),(3,6,7,12,13,14),(3,6,8,9,11,16),(4,5,7,10,13,16),(4,5,8,11,12,14)$, $(5,6,9,10,13,14)\}, \ldots$,

$S_{11}^{\prime}=\{(1,2,3,6,7,10), \quad(1,2,4,5,8,9), \quad(1,3,4,11,12,13), \quad(1,5,6,11,14,15), \quad(1,7,8,12,14,16)$, $(1,9,10,13,15,16),(2,3,4,14,15,16),(2,5,6,12,13,16),(2,7,8,11,13,15),(2,9,10,11,12,14)$, $(3,6,8,9,13,14),(3,7,9,10,11,16),(3,5,8,10,12,15),(4,6,7,9,12,15),(4,6,8,10,11,16)$, $(5,5,7,10,13,14)\}$ 。 
As the number of different configurations for design $(16,10,6)$ equals $S_{(i)}^{\prime}=\left\{B_{1}^{(1)}, B_{2}^{(1)}, \cdots, B_{16}^{(1)}\right\}$ of design $(16,6,2)$, so the steps for constructing other design of $(16,10,6) S_{1}^{\prime \prime}, S_{2}^{\prime \prime}, \ldots, S_{11}^{\prime \prime}$ are as follows:

(1) Give the16 blocks of first design $S_{1}^{\prime}=\left\{B_{1}^{(1)}, B_{2}^{(1)}, \cdots, B_{16}^{(1)}\right\}$ from $(16,6,2)$, then use complementary relationship between the blocks of residual design $S^{\prime \prime}=\left\{B_{1}^{(2)}, B_{2}^{(2)}, \cdots, B_{16}^{(2)}\right\}$ from $(16,10,6)$ and blocks of export design $S^{\prime}=\left\{B_{1}^{(1)}, B_{2}^{(1)}, \cdots, B_{16}^{(1)}\right\}$ from $(16,6,2)$ :

$B_{1}^{(2)}=X \cap B_{1}^{(1)}, \quad B_{2}^{(2)}=X \cap B_{2}^{(1)}, \quad \ldots, \quad B_{16}^{(2)}=X \cap B_{16}^{(1)}, \quad$ get the first residual design $S_{1}^{\prime \prime}=\left\{B_{1}^{(2)}, B_{2}^{(2)}, \cdots, B_{16}^{(2)}\right\}$ as follows:

$S_{1}^{\prime \prime}=\{(7,8,9,10,11,12,13,14,15,16), \quad(3,4,5,6,11,12,13,14,15,16)$,

$(2,4,5,6,8,9,10,14,15,16), \quad(2,3,5,6,7,9,10,12,13,16), \quad(2,3,4,6,7,8,10,11,13,15)$,

$(2,3,4,5,7,8,9,11,12,14),(1,4,5,6,8,9,10,11,12,13),(1,3,5,6,7,9,10,11,14,15)$,

$(1,3,4,6,7,8,10,12,14,16),(1,3,4,5,7,8,9,13,15,16), \quad(1,2,5,6,7,8,11,12,15,16)$,

$(1,2,4,6,7,9,12,13,14,15), \quad(1,2,4,5,7,10,11,13,14,16), \quad(1,2,3,6,8,9,11,13,14,16)$,

$(1,2,3,5,8,10,12,13,14,15), \quad(1,2,3,4,9,10,11,12,15,16)\}$

(2) List the complementary relationship in turn between the second, the third design from $(16,10,6)$ and 16 blocks from design $(16,10,6)$ as follows:

$S_{2}^{\prime \prime}=\{(3,4,9,10,11,12,13,14,15,16), \quad(5,6,7,8,11,12,13,14,15,16)$,

$(2,4,6,7,8,9,10,14,15,16),(2,3,5,7,8,9,10,12,13,16),(2,3,4,5,6,8,10,11,13,15)$,

$(2,3,4,5,6,7,9,11,12,14),(1,4,6,7,8,9,10,11,12,13),(1,3,5,7,8,9,10,11,14,15)$,

$(1,3,4,5,6,8,10,12,14,16),(1,3,4,5,6,7,9,13,15,16), \quad(1,2,5,6,10,11,12,13,14,16)$,

$(1,2,4,5,8,9,10,11,13,16), \quad(1,2,4,5,7,10,12,13,14,15), \quad(1,2,3,6,8,9,11,12,14,15)$,

$(1,2,3,6,7,9,10,13,15,16), \quad(1,2,3,4,7,8,11,15,16)\}, \ldots$,

$S_{11}^{\prime \prime}=\{(4,5,8,9,11,12,13,14,15,16), \quad(3,6,7,10,11,12,13,14,15,16)$,

$(2,5,6,7,8,9,10,14,15,16), \quad(2,3,4,7,8,9,10,12,13,16), \quad(2,3,4,5,6,9,10,11,13,15)$,

$(2,3,4,5,6,7,8,11,12,14),(1,5,6,7,8,9,10,11,12,13),(1,3,4,7,8,9,10,11,14,15)$,

$(1,3,4,5,6,9,10,12,14,16), \quad(1,3,4,5,6,7,8,13,15,16), \quad(1,2,4,5,7,10,11,12,15,16)$,

$(1,2,4,6,8,10,12,13,14,15), \quad(1,2,4,6,7,9,11,13,14,16), \quad(1,2,3,5,8,10,11,13,14,16)$,

$(1,2,3,5,7,9,12,13,14,15),(1,2,3,6,8,9,11,12,15,16)\}, \ldots$.

\section{Conclusions}

Main contribution of this paper: (1) Illustrates the complementary relationship and nature of the export design $(v, k, \lambda)$ and the residual design $(v, v-k, v-2 r+\lambda)$.(2) Propose the design and construction method of $(v, k, \lambda)$ and $(v, v-k, v-2 r+\lambda)$;(3) Construct design $S_{1}^{\prime}, S_{2}^{\prime}, \cdots \cdots, S_{11}^{\prime}$ from $(16,6,2)$ and 11 different configured design $S_{1}^{\prime \prime}, S_{2}^{\prime \prime}, \cdots \cdots, S_{11}^{\prime \prime}$ from $(16,10,6)$.

\section{References}

[1]Wan-di Wei, Combinatorial Theory [M]. Bei Jing: Science Press, 1987. 
[2] D. R. Hughes, F. C. Diper. Design Theory [M]. London: Cambridge University Press, 1985.

[3] Fred S. Roberts, Barry Tesman. Applied Combinatory [M]. Beijing: China Machine Press, 1984.

[4] J. H. Vanlint, R. M. Wilson. A Course in Combinatory [M]. Beijing: China Machine Press, 1992.

[5] Richard A. Brualdi. Introductory Combinatory [M]. Beijing: China Machine Press, 1999.

[6] CHOU Wan-xi. A Method of Constructing Kirkman Triple System of Order $k \times t$ [J]. Mathematics In Practice and Theory, 2004, 34(9):144-150.

[7] CHOU Wan-xi. Seiner Triple System and Its Construction Met [J]. Journal of Anhui University of Science and Technology(Natural Science), 2004, 24(3):76-80.

[8] Ai Mingyao, Zhang Runchu. UNIFORMAITY OF BLOCK DESIGNS [J]. Journal of Nankai University (Natural Science), 2003, 36(2):89-92.

[9] Sheng Lin ZHOU. The Ree Groups $2 \mathrm{G} 2$ (q) and 2-(v,k, 1) Block Designs (II)[J]. Acta Mathematica Sinica, 2003,46(4):823-828.

[10] LIU Jian-guo, GUO Qiang, XIA Zun-quan. Optimal Block Designs in Mixed Effects Models [J]. Mathematics in Practice and Theory, 2005, 35(5):97-103.

[11] HAN Gang, LI Hui-ling. Block designs admitting an automorphism group with an alternating socle[J]. Journal of Zhejiang University (Sciences Edition), 2003, 29(3):241-245.

[12] LIU Wei- jun, MA Chuan-gui.On some theor ems of block designs [J].Journal of Zhejiang University(Science Edition),2000,27(4):361- 363.

[13] TIAN Jin-ting,ZHANG Ying-shan,ZHANG Xiao-qin,PAN Chang-yuan,GAN Yuan-yuan. The Comparison and Application of Balanced Block Orthogonal Arrays and Orthogonal Arrays [J]. Mathematics in Practice and Theory, 2009, 39 (22) :59

[14] J.X. Yin, C.M. Wang, Kirkman covering designs with even-sized holes, Discrete Mathematics [J]. 2009, 309(6):1422-1434.

[15] ZHANG Ming-zhu, WU Ya-zhen,ZHANG Jian-jun. Replacement Construction of Orthogonal Balanced Block Designs[J]. Journal of Shanxi University:Natural Science Edition,

$2012 \quad-435(.03): 472$

[16] X. Y. Li, Z. D. Xu, and W. X. Chou, A method of constructing Kirkman triple system of higher order, in proceeding of Control and Decision Conference (CCDC), 2318-2322, 2010.

[17] X. Y. Li, Z. D. Xu, and W. X. Chou, A new method of constructing Steiner triple systems, in proceeding of Control and Decision Conference (CCDC), 3760-3764, 2010.

[18] H. Cao, Y. Tang, On Kirkman packing designs KPD (\{3,4\},v), Discrete Mathematics, Vol. 279, No. 1-3, 121-133, 2004.

[19] L.J. Ji, J.G. Lei, Further results on large sets of Kirkman triple systems, Discrete Mathematics, Vol. 308, No. 20, 4643-4652, 2008.

[20] H. Peter, on the chromatic number of Steiner triple systems of order 25, Discrete Mathematics, Vol. 299, No. 1-3, 120-128, 2005. 